\title{
Non-Linear Effect of Volume Fraction of Inclusions on the Effective Thermal Conductivity of Composite Materials: A Modified Maxwell Model
}

\author{
Sajjan Kumar ${ }^{1}$, Rajpal Singh Bhoopal ${ }^{1}$, Pradeep Kumar Sharma ${ }^{1}$, Radhey Shyam Beniwal ${ }^{2}$, \\ Ramvir Singh $^{\mathbf{1}^{*}}$ \\ ${ }^{1}$ Heat Transfer Laboratory, Department of Physics, University of Rajasthan, Jaipur, India \\ ${ }^{2}$ National Institute of Science Communication and Information Resources, CSIR, New Delhi, India \\ E-mail:"singhrvs@rediffmail.com \\ Received August 6, 2011; revised September 13, 2011; accepted September 23, 2011
}

\begin{abstract}
In this paper, non-linear dependence of volume fraction of inclusions on the effective thermal conductivity of composite materials is investigated. Proposed approximation formula is based on the Maxwell's equation, in that a non-linear term dependent on the volume fraction of the inclusions and the ratio of the thermal conductivities of the polymer continuum and inclusions is introduced in place of the volume fraction of inclusions. The modified Maxwell's equation is used to calculate effective thermal conductivity of several composite materials and agreed well with the earlier experimental results. A comparison of the proposed relation with different models has also been made.
\end{abstract}

Keywords: Effective Thermal Conductivity, Empirical Correction Term, Composite Materials

\section{Introduction}

Theoretical prediction of effective thermal conductivity (ETC) for multi-phase composite materials is very useful not only for analysis and optimization of the material performance, but also for new material designs. The correct modeling for thermal coefficients of these materials has a great value due to their excellent thermal and mechanical properties and their use in industrial applications and technological fields. The challenges in modeling complex materials come mainly from the inherent variety and randomness of their internal microstructures, and the coupling between the components of different phases. In literature, several attempts have been made to develop expressions for effective thermal conductivity of two-phase materials by various researchers such as, Maxwell [1], Lewis and Nielsen [2], Cunningham and Peddicord [3], Torquato [4], Hadley [5], Agari and Uno [6], Misra et al. [7], Singh and Kasana [8], and Verma et al. [9]. Lewis and Nielsen [2] reported a semi-empirical model incorporating the effect of the shape and the orientation of particles or, the type of packing for a two-phase system. Other approach for a thermal conductivity prediction was initiated by Torquato [4] for dis- persed spherical or cylinder particles. This approach also takes into account the filler geometry and the statistical perturbation around each filler particle. Agari and Uno [6] also proposed another semi-empirical model, which is based on the argument that the enhanced thermal conductivity of highly filled composites originates from forming conductive chains of fillers. Verma et al. [9] developed a porosity dependence correction term for spherical and non-spherical particles. Calmidi and Mahajan [10] presented a one-dimensional heat conduction model, considering the porous medium to be formed of two-dimensional array of hexagonal cells. Bhattacharya et al. [11] extended the analysis of Calmidi and Mahajan for metal foams of a complex array of interconnected fibers with an irregular lump of metal at the intersection of two fibers. Pabst and Gregorova [12] developed a simple second-order expression for the porosity dependence of thermal conductivity.

In this study, a non-linear second-order correction term is developed in place of volume fraction of inclusions and used in the Maxwell's model [1] for estimation of ETC of metal filled composite materials. Originally, Maxwell's model was derived for low dispersion of filler particles in the matrix. Here, a non-linear second-order 
empirical expression in place of filler volume fraction has been proposed and the unknown coefficients have been determined using boundary conditions and experimental results reported earlier. Volume fraction of inclusions in the Maxwell's model is then replaced by the non-linear second-order correction term. The results obtained using modified Maxwell's model show a better agreement with experimental values.

\section{Mathematical Formulation}

By solving Laplace's equation and assuming absence of any interactions between the filler particles, Maxwell [1] calculated the effective thermal conductivity (ETC) of a random distribution of spheres in a continuous medium for low filler concentrations as:

$$
k_{e}=k_{m} \frac{k_{f}+2 k_{m}+2 \varphi\left(k_{f}-k_{m}\right)}{k_{f}+2 k_{m}-\varphi\left(k_{f}-k_{m}\right)}
$$

Where $k_{e}, k_{m}$ and $k_{f}$ are effective thermal conductivity, matrix thermal conductivity and thermal conductivity of fillers, respectively, and $\varphi$ is the volume fraction of inclusions. This model was developed for low dispersions i.e. for lower volume fraction of filler phase. Maxwell's model fails to predict ETC of composite materials having higher volume fraction metallic inclusions. In composite materials, the inclusions most frequently used are particles of carbon, aluminum, copper, iron, silicon, brass, graphite and magnetite, respectively. Therefore, to predict ETC of composite materials some correction is needed in the Maxwell's model. This correction may be in thermal conductivity of the constituent phases or in the fractional volume of the constituents.

Pabst and Gregorova [12] developed a model, which shows the non-linear porosity dependent thermal conductivity of two-phase materials. Verma et al. [9] have also developed a model for ETC of two-phase materials with spherical and non-spherical inclusions using a correction term. Some experimental results [13-18] also show the non-linear dependence of ETC on the volume fraction of filler phase. On reviewing all these facts, we concluded that there should be a non-linear correction term in place of volume fraction of inclusions in dissimilar materials. Therefore, keeping in mind the above facts, we assumed a non-linear second-order correction term in place of volume fraction of inclusions as:

$$
F_{p}=\alpha \varphi+\beta \varphi^{2}
$$

Here $\alpha$ and $\beta$ are empirical constant obeying the following boundary condition as:

(1) When $\varphi \rightarrow 0$ then $F_{p} \rightarrow 0$

and

(2) When $\varphi \rightarrow 1$ then $F_{p} \rightarrow 1$
From Equation (2), condition (1) is satisfied and to satisfy condition (2) constants $\alpha$ and $\beta$ should have the following relation:

$$
\alpha+\beta=1
$$

By using Equation (3)

$$
F_{p}=\alpha \varphi+(1-\alpha) \varphi^{2}
$$

Therefore, on replacing volume fraction of inclusions $\varphi$ by correction term $F_{p}$ in Maxwell's Equation (1), the expression for ETC becomes

$$
k_{e}=k_{m} \frac{k_{f}+2 k_{m}+2 F_{p}\left(k_{f}-k_{m}\right)}{k_{f}+2 k_{m}-F_{p}\left(k_{f}-k_{m}\right)}
$$

Using this relation, we have calculated ETC of several samples like high-density polyethylene (HDPE), polypropylene (PP) filled with metal particles, epoxy resin filled with $\mathrm{SiO}_{2}, \alpha-\mathrm{Al}_{2} \mathrm{O}_{3}, \mathrm{AlN}$ and sandstones filled with air, n-Hepten, and water respectively.

\section{Results and Discussion}

The value of empirical constant $\alpha$ is found to depend upon ratio of thermal conductivity of the constituents, size, shape and distribution of filler particles in the matrix and therefore have different values for different type of materials. To determine $\alpha$, curve-fitting method was applied for various samples using data reported earlier [13-18] and found that the expression for $\alpha$ comes out to be:

$$
\alpha=A\left(\frac{k_{f}}{k_{m}} \varphi^{1 / 3}\right)+B
$$

Here $A$ and $B$ are slope and intercept of the curves for various samples. Optimized values of these constants have been used in such a way that, it should have consistency with the boundary conditions (1) and (2). The values of $A$ and $B$ of various samples computed using relation (6) are shown in Table 1.

To validate modified Maxwell's relation (5), several samples of HDPE and PP filled with metal particles, epoxy resin filled with $\mathrm{SiO}_{2}, \alpha-\mathrm{Al}_{2} \mathrm{O}_{3}$ and AIN and sandstones filled with air, n-Hepten, and water with increasing filler concentration have been taken in the present computations. For calculations purpose, input parameters have been used from the results published earlier [13-18]. The values of ETC for various samples are calculated using modified Maxwell's relation (5) and the comparison of predicted values and experimental results are shown in Figures 1-15.

The results for HDPE filled with metal particle are shown in Figures 1-4. It is seen from the Figures that models $[1,2]$ predicts higher value of ETC at lower filler concentration where as our model have better predictions, but at higher filler concentration, our model predict little 
Table 1. Value of $A$ and $B$ for different samples.

\begin{tabular}{cccc}
\hline S. No. & Sample & $\mathrm{A}$ & $\mathrm{B}$ \\
\hline 1. & $\mathrm{HDPE} / \mathrm{Sn}$ & 0.03171 & -0.73279 \\
2. & $\mathrm{HDPE} / \mathrm{Zn}$ & 0.03008 & -3.03067 \\
3. & $\mathrm{HDPE} / \mathrm{Cu}$ & 0.01028 & -3.69046 \\
4. & $\mathrm{HDPE} / \mathrm{Fe}$ & 0.03143 & -1.89218 \\
5. & $\mathrm{HDPE} / \mathrm{Si}$ & 0.00417 & 0.71951 \\
6. & $\mathrm{PP} / \mathrm{Al}_{1}$ & 0.00372 & -1.12155 \\
7. & $\mathrm{PP} / \mathrm{Al}_{2}$ & 0.00294 & -0.21239 \\
8. & Epoxy resin $/ \mathrm{SiO}_{2}$ & -0.012339 & 2.201449 \\
9. & Epoxy resin $/ \alpha-\mathrm{Al}_{2} \mathrm{O}_{3}$ & 0.0007188 & 1.773956 \\
10. & Epoxy resin $/ \mathrm{AlN}$ & -0.002051 & 3.397643 \\
11. & $\mathrm{HDPE} / \mathrm{Al}_{2} \mathrm{O}_{3}$ & 0.009658 & 1.437955 \\
12. & $\mathrm{HDPE} / \mathrm{Bronze}$ & 0.073825 & 3.910710 \\
13. & $\mathrm{Sandstone} / \mathrm{Air}$ & -2031.33973 & 7.51613 \\
14. & $\mathrm{Sandstone} / \mathrm{n}-\mathrm{Hepten}$ & -179.06678 & 4.28328 \\
15. & $\mathrm{Sandstone} / \mathrm{Water}$ & -28.56255 & 3.27327 \\
\hline
\end{tabular}

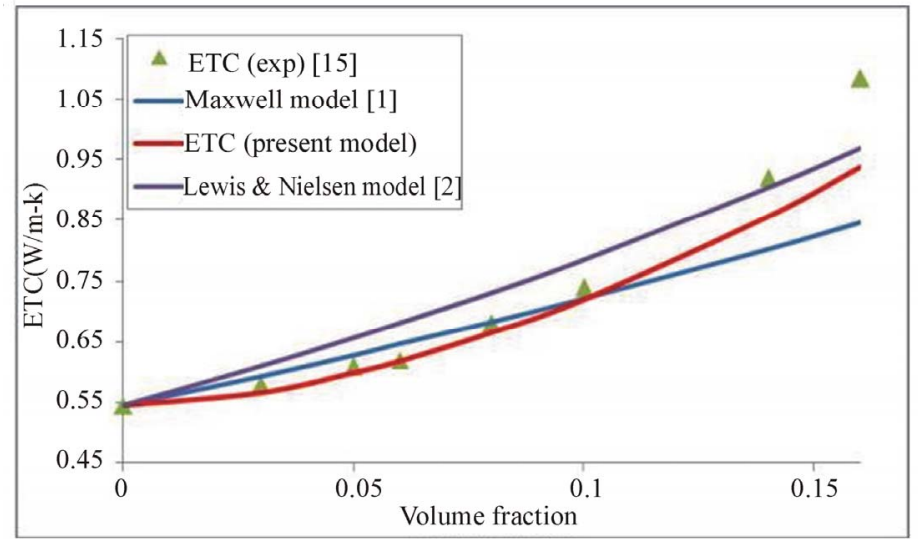

Figure 1. Comparisons of experimental and predicted values of ETC; HDPE filled with Tin.

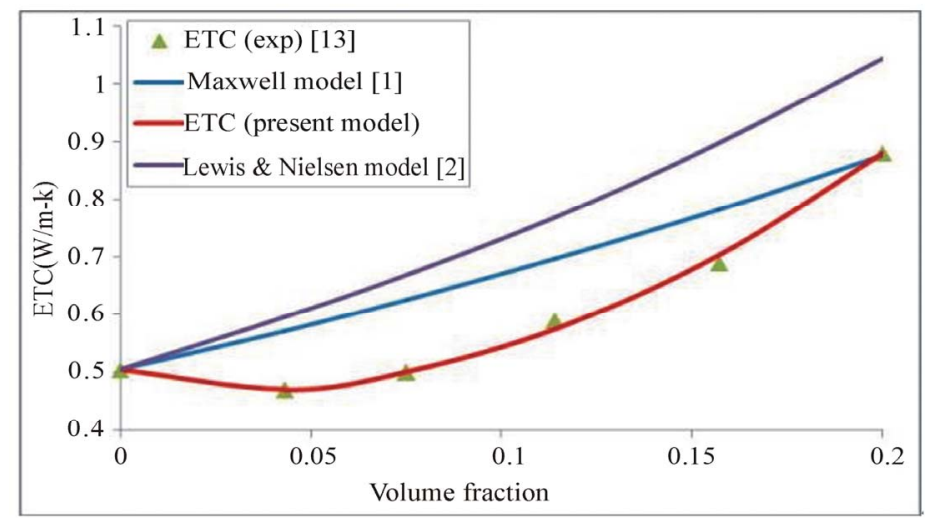

Figure 2. Comparisons of experimental and predicted values of ETC; HDPE filled with Zinc.

lower values of ETC. Figures 2-4 show that the values of ETC calculated by most of the existing models are higher but our model predicts fairly well in the whole range of filler volume fractions.

It has been observed from the Figures 5-7 that ETC have a rapid increment when volume fraction of filler phase is increased. Here filler phase has higher thermal conductivity than the matrix phase. At lower volume fractions, filler particles are randomly scattered in the matrix phase but when volume fraction of inclusions 


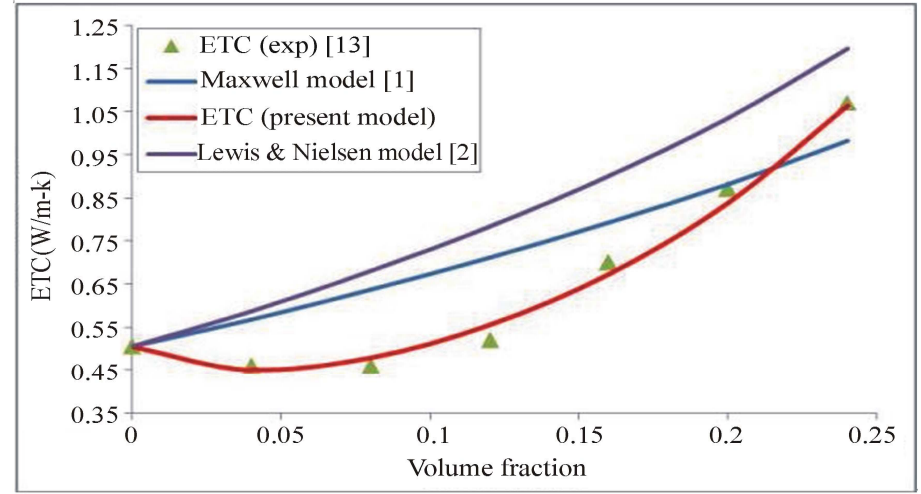

Figure 3. Comparisons of experimental and predicted values of ETC; HDPE filled with Copper.

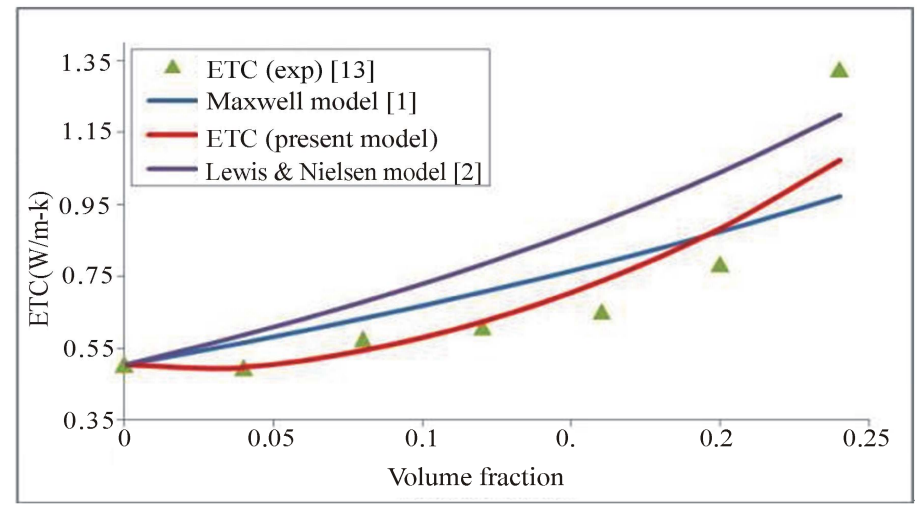

Figure 4. Comparisons of experimental and predicted values of ETC; HDPE filled with Iron.

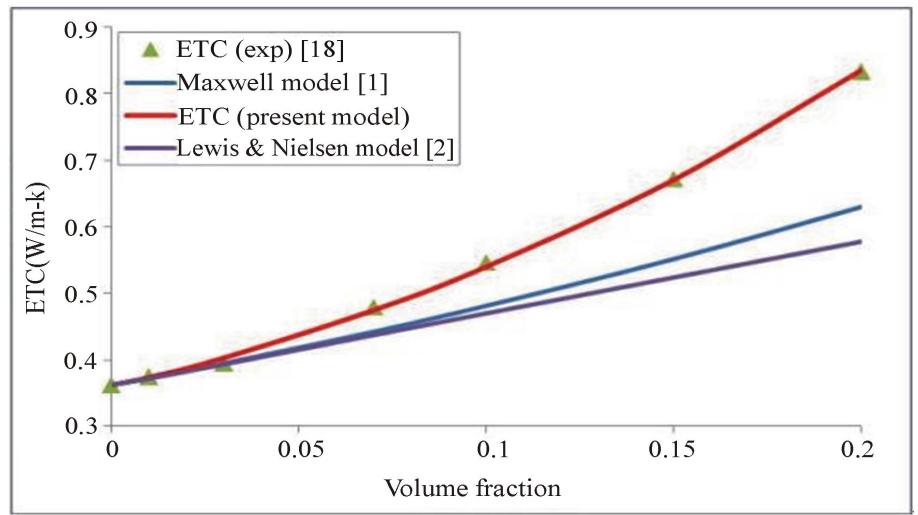

Figure 5. Comparisons of experimental and predicted values of ETC; HDPE filled with Silicon.

increases, then particles begin to touch each other and form conductive chains in the direction of heat flow, due to this ETC increases rapidly. Probability of forming conductive chains is higher in the case of smaller particles. Slightly oxidized aluminum particles for the preparation of $\mathrm{PP} / \mathrm{Al}$ samples [14] were used and the thermal conductivity value used for our computations of ETC is the one given for pure heavy aluminum. In reality, the thermal conductivity of the fillers used in this computation is probably lower than this value, and depends on the mean size of the particles. Therefore, at higher concentration of filler particles, modified Maxwell's model predicts higher value of ETC then the experimental results and larger deviation occur. However, the model predicts fairly well up to $50 \%$ of filler concentration.

The results for Epoxy resin and HDPE filled with ox- 


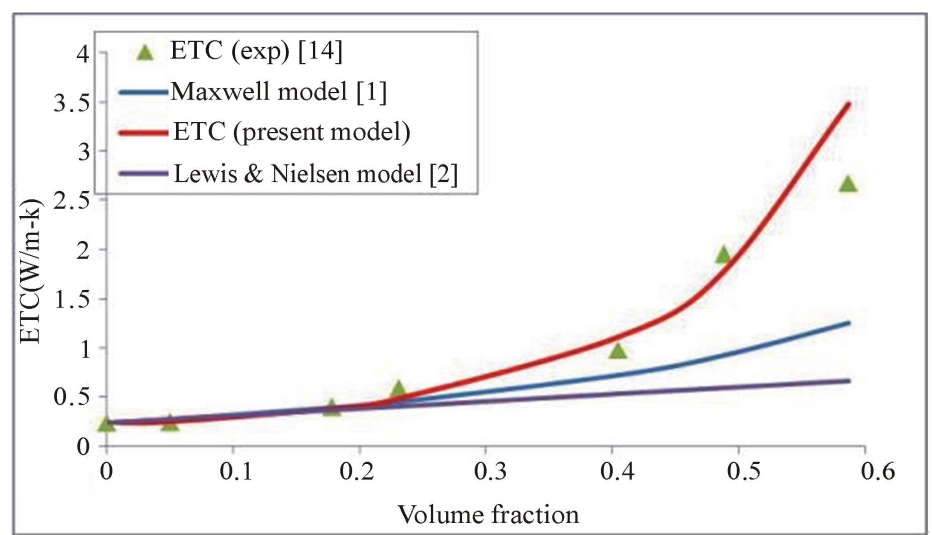

Figure 6. Comparisons of experimental and predicted values of ETC; Polypropylene filled with Aluminum (mean diameter of $8 \mu \mathrm{m})$.

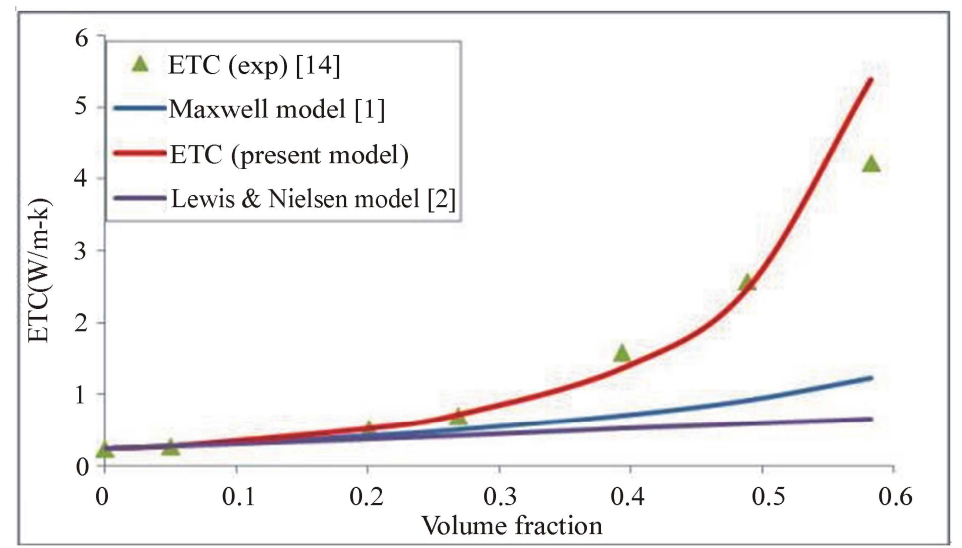

Figure 7. Comparisons of experimental and predicted values of ETC; Polypropylene filled with Aluminum, (mean diameter of $44 \mathrm{\mu m}$ ).

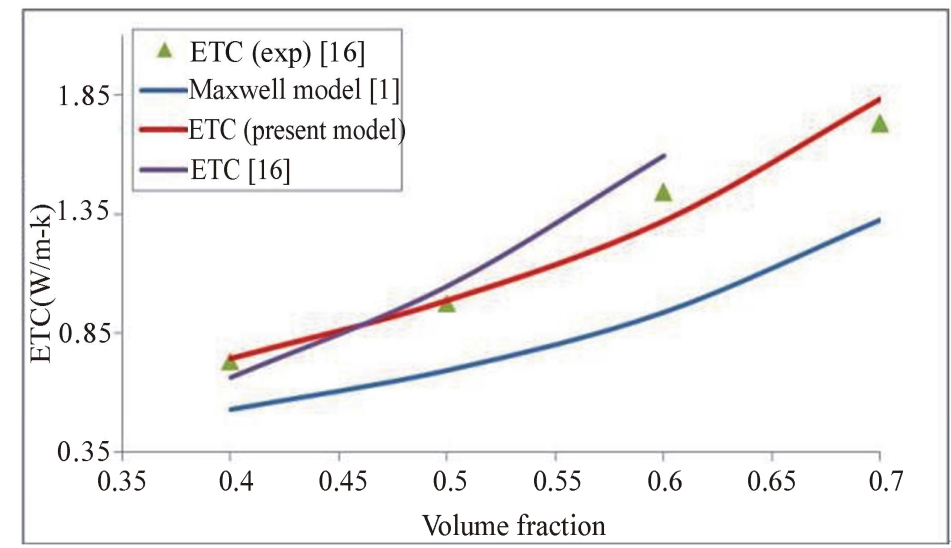

Figure 8. Comparisons of experimental and predicted values of ETC; Epoxy resin filled with $\mathrm{SiO}_{2}$.

ides are shown in Figures 8-12. It is observed from Figures 8-10 that Maxwell's model [1] calculate lower value of ETC but modified Maxwell's Equation (5) predict better value than [16]. Figure 11 shows that the models $[1,2]$ predict lower value of ETC but our model prediction have better agreement and it is also observed from Figure 12.

The results for ETC of sandstone filled with air, 


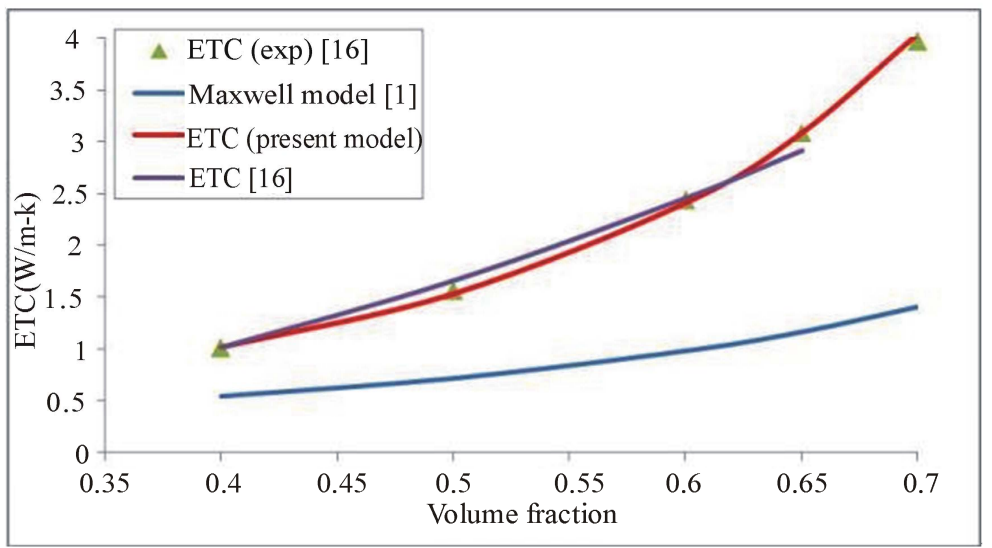

Figure 9. Comparisons of experimental and predicted values of ETC; Epoxy resin filled with $\mathrm{Al}_{2} \mathrm{O}_{3}$.

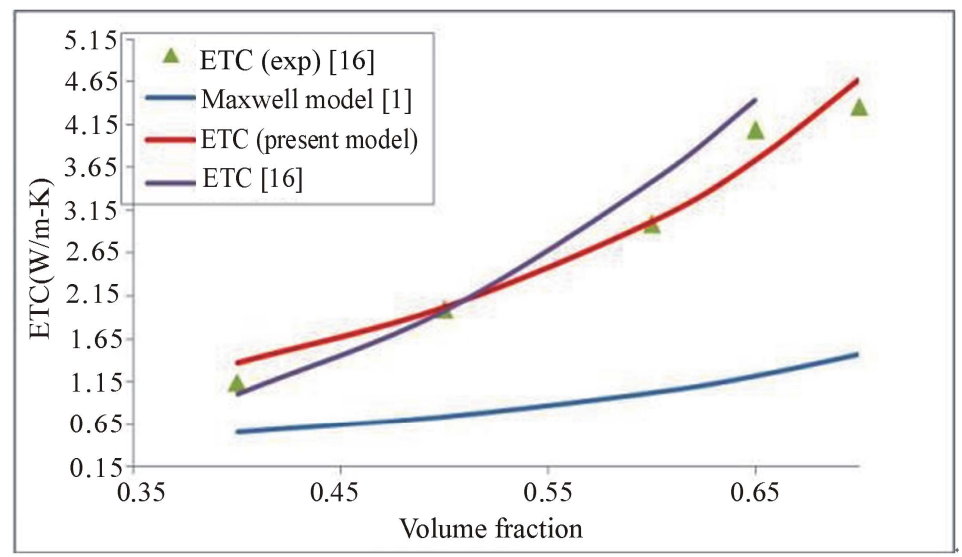

Figure 10. Comparisons of experimental and predicted values of ETC; Epoxy resin filled with AIN.

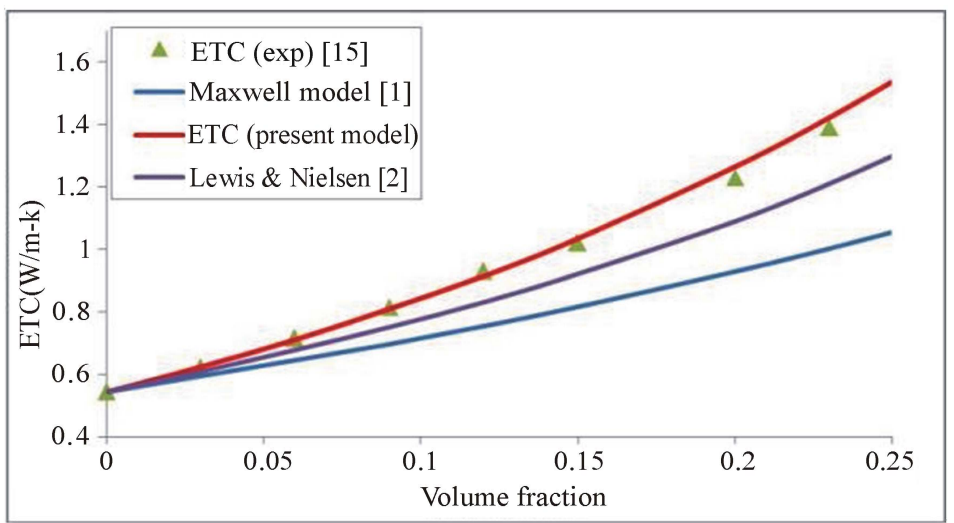

Figure 11. Comparisons of experimental and predicted values of ETC; HDPE filled with Aluminum Oxide.

n-Hepten and water are shown in Figures 13-15. We note from these Figures that ETC decreases as the volume fraction of filler phase increases due to the lower value of thermal conductivity of filler phase. Models [1,2] observe this effect but they predict higher values of ETC. It is noticed from these Figures that modified Maxwell's model have better agreement with the experimental results [17].

At low filler volume content, up to $15 \%$, a moderate increase in thermal conductivity was observed in the earlier results. We noticed that in this region, most of the predictive models of thermal conductivity are applicable 


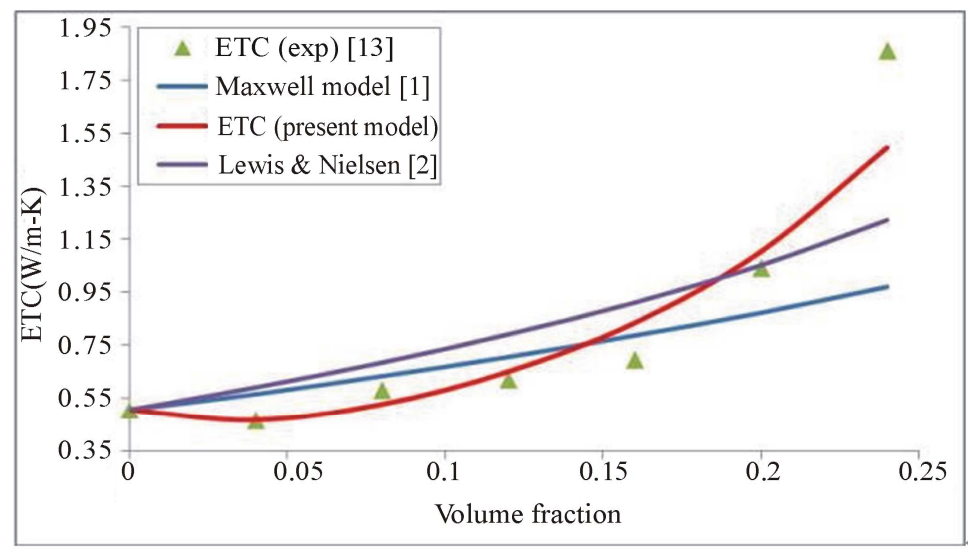

Figure 12. Comparisons of experimental and predicted values of ETC; HDPE filled with Bronze.

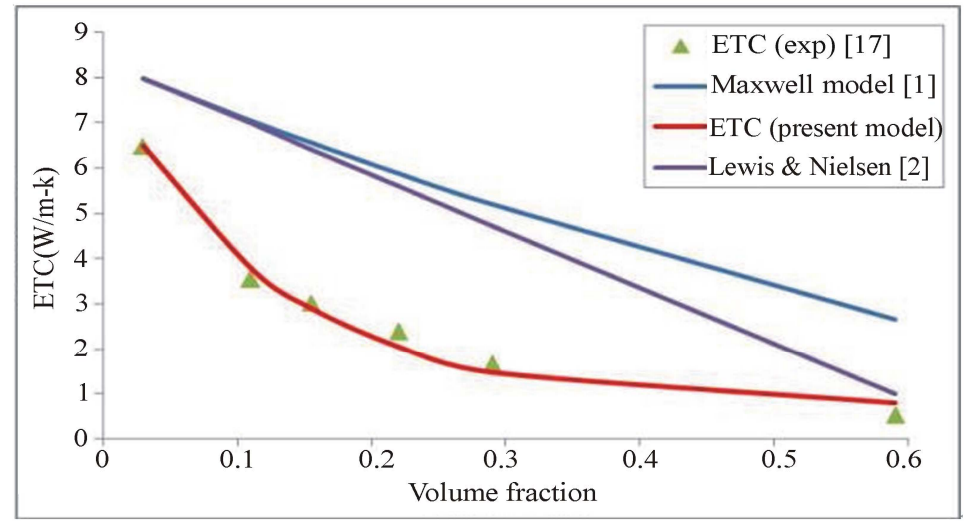

Figure 13. Comparisons of experimental and predicted values of ETC; Sandstones filled with Air.

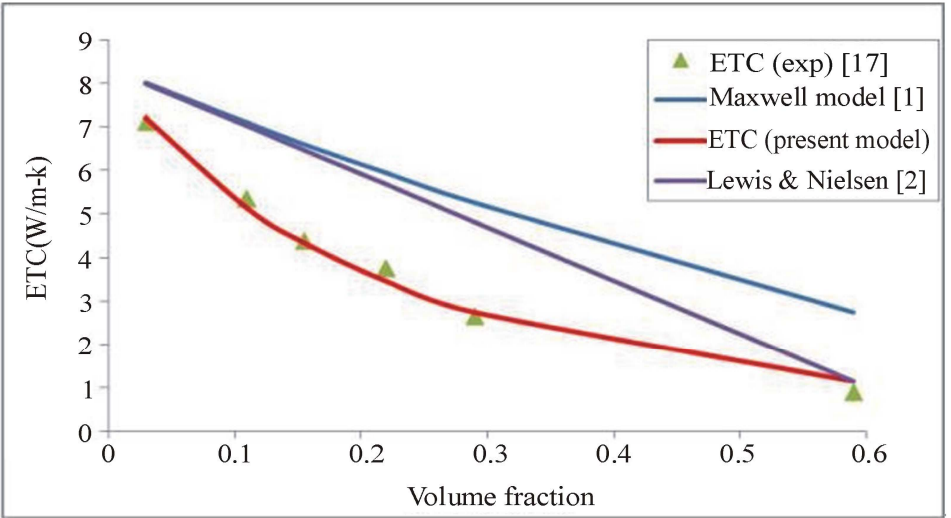

Figure 14. Comparisons of experimental and predicted values of ETC; Sandstones filled with n-Hepten.

on composite materials. For more heavily metal filled composites, a non-linear increase in thermal conductivity was observed and almost all the models fail to predict ETC in this region. Because most of the theoretical models do not consider the size, shape and distribution of filler particles in the matrix and at higher filler content, the filler particles tend to form agglomerates due to it conductive chains form, resulting in a rapid increase in thermal conductivity.

\section{Conclusions}

In the present paper, it is concluded that there is a linear variation in ETC when filler particles approaches up to 


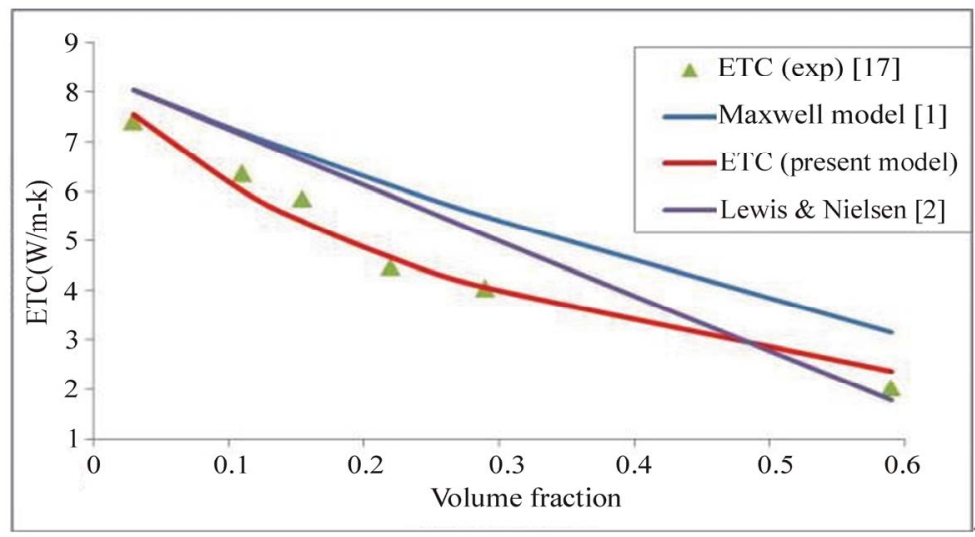

Figure 15. Comparisons of experimental and predicted values of ETC; Sandstones filled with Water.

$15 \%$ by volume in the matrix. Non-linearity occurs when filler content increases from $15 \%$ by volume in most of the materials having high thermal conductivity ratio of the constituents. The present relation (5) has a constant $\alpha$, which may depend on various factors of the materials like fractional volume of inclusions, conductivity ratio of the constituents, size, shape and distribution of inclusions and therefore have different values for a variety of materials. We note that the distribution of inclusions in the matrix has strong implications on ETC of composite materials. Nearly all theoretical models assume homogeneous dispersion in the matrix but it is not true for most of the complex materials.

We also noticed that the expression derived for $F_{p}$ using the concept of non-linearity works well for a variety of materials like HDPE and PP filled with metal particle, epoxy resin filled with $\mathrm{SiO}_{2}, \alpha-\mathrm{Al}_{2} \mathrm{O}_{3}$ and $\mathrm{AlN}$ and sandstones filled with air, n-Hepten and water. It is also concluded that whatever an approach is used a correction term is always needed to predict correct values of ETC of randomly mixed real systems. It is always present in the models in one form or the other. We have also reached at the conclusion that in most of the models, correction terms are of non-linear in nature when conductivity ratio of the constituents is high.

\section{Acknowledgements}

A Junior Research Fellowship awarded by CSIR to one of the authors (SK) is gratefully acknowledged.

\section{References}

[1] J. C. Maxwell, "A Treatise on Electricity and Magnetism," 3rd Edition, Clarendon Press, Oxford, 1904.

[2] T. Lewis and L. Nielsen, "Dynamic Mechanical Properties of Particulate-Filled Composite," Journal of Applied Polymer Science, Vol. 14, No. 6, 1970, pp. 1449-1471. doi:10.1002/app.1970.070140604

[3] M. E. Cunningham and K. L. Peddicord, "Heat Conduction in Spheres Packed in an Infinite Regular Cubical Array," International Journal of Heat and Mass Transfer, Vol. 24, No. 7, 1981, pp. 1081-1088. doi:10.1016/0017-9310(81)90157-5

[4] S. Torquato, "Effective Electrical Conductivity of TwoPhase Disordered Composite Media," Journal of Applied Physics, Vol. 58, No. 10, 1985, pp. 3790-3797. doi: $10.1063 / 1.335593$

[5] G. R. Hadley, "Thermal Conductivity of Packed Metal Powers," International Journal of Heat and Mass Transfer, Vol. 29, No. 6, 1986, pp. 909-920. doi:10.1016/0017-9310(86)90186-9

[6] Y. Agari and T. Uno, "Estimation on Thermal Conductivities of Filled Polymers," Journal of Applied Polymer Science, Vol. 32, No. 7, 1986, pp. 5705-5712. doi:10.1002/app.1986.070320702

[7] K. Misra, A. K. Shrotriya, R. Singh and D. R. Chaudhary, "Porosity Correction for Thermal Conduction in Real Two-Phase Systems," Journal of Physics D: Applied Physics, Vol. 27, No. 4, 1994, pp. 732-735. doi: 10.1088/0022-3727/27/4/009

[8] R. Singh and H. S. Kasana, "Computational Aspects of Effective Thermal Conductivity of Highly Porous Metal Foams," Applied Thermal Engineering, Vol. 24, No. 13, 2004, pp. 1841-1849.

doi:10.1016/j.applthermaleng.2003.12.011

[9] L. S. Verma, A. K. Shrotriya, R. Singh and D. R. Chaudhary, "Thermal Conduction in Two-Phase Materials with Spherical and Non-Spherical Inclusion," Journal of Physics D: Applied Physics, Vol. 24, No. 10, 1991, pp. 17291737. doi:10.1088/0022-3727/24/10/006

[10] V. V. Calmidi and R. L. Mahajan, "The Effective Thermal Conductivity of High Porosity Fibrous Metal Foam," ASME Journal of Heat Transfer, Vol. 121, No. 2, 1999, pp. 466-471. doi:10.1115/1.2826001

[11] A. Bhattacharya, V. V. Calmidi and R. L. Mahajan, "Thermoplastic Properties of High Porosity Metal Foam," International Journal of Heat and Mass Transfer, Vol. 45, 2002, pp. 1017-1031. 


\section{doi:10.1016/S0017-9310(01)00220-4}

[12] W. Pabst and E. Gregorova, "A New Percolation- Threshold Relation for the Porosity Dependence of Thermal Conductivity," Ceramics International, Vol. 32, No. 1, 2006, pp. 89-91. doi:10.1016/j.ceramint.2004.12.007

[13] N. M. Sofian, M. Rusu, R. Neagu and E. Neagu, "Metal Powder-Filled Polyethylene Composites V. Thermal Properties," Journal of Thermoplastic Composite Materials, Vol. 14, 2001, pp. 20-33. doi:10.1106/9N6K-VKH1-MHYX-FBC4

[14] A. Boudenne, L. Ibos, M. Fois, E. Gehin and J. C. Majeste, "Thermophysical Properties of Polypropylene/ Aluminum Composites," Journal of Polymer Science Part B: Polymer Physics, Vol. 42, No. 4, 2004, pp. 722-732. doi:10.1002/polb.10713

[15] D. Kumlutas and I. H. Tavman, "A Numerical and Experimental Study on Thermal Conductivity of Particle
Filled Polymer Composite," Journal of Thermoplastic Composite Materials, Vol. 19, 2006, pp. 441-455. doi:10.1177/0892705706062203

[16] S. W. Kim, B. Choi, S. H. Lee and K. H. Kang, "Measurement of Thermophysical Properties of Articulate- Filled Polymer Composites," High Temperature-High Pressures, Vol. 37, 2008, pp. 21-30.

[17] W. Woodside and J. H. Messmer, "Thermal Conductivity of Porous Media II Consolidated Rocks," Journal of Applied Physics, Vol. 32, No. 9, 1961, pp. 1699-1706. doi: $10.1063 / 1.1728420$

[18] T. K. Dey and M. Tripathi, "Thermal Properties of Silicon Powder Filled High-Density Polyethylene Composites," Thermochimica Acta, Vol. 502, No. 1-2, 2010, pp 35-42. doi:10.1016/j.tca.2010.02.002 